Gynäkologische Endokrinologie 2009 · 7:274-274

DOI 10.1007/s10304-009-0338-1

Online publiziert: 25. Oktober 2009

(c) Springer Medizin Verlag 2009

\section{Redaktion}

R. Felberbaum, Kempten

C. Blume · K. Geue · S. Waczek · G. Allgayer · C. Daufratshofer · R. Felberbaum Klinik für Frauenheilkunde und Geburtshilfe,Perinatalzentrum, Zentrum für Reproduktionsmedizin, Klinikum Kempten - Oberallgäu, Kempten (Allgäu)

\title{
Triamniote-dichoriale Drillingsgravidität
}

\section{Epikrise}

25-jährige I-Grav/o-Para; Zustand nach IVF mit ICSI extra muros nach kontrollierter ovarieller Hyperstimulation im langen Protokoll (GnRH-Agonistenprotokoll) bei primärer andrologisch bedingter Ehesterilität. Nach Transfer von 2 Embryonen Entwicklung eines schweren, hospitalisationspflichtigen ovariellen Überstimulationssyndroms $\left(\mathrm{OHSS} \mathrm{III}{ }^{\circ}\right)$. Bei regelrecht ansteigenden HCG-Werten Feststellung einer Drillingsgravidität in der rechnerisch 6. SSW. In der 10. SSW eindeutige Darstellung einer triamnioten-dichorialen Drillingsgravidität. T-Sign und Lambda-Sign können so in einer Gravidität gleichzeitig beobachtet werden. Einer der rückgesetzten Embryonen hat sich spontan geteilt.

\section{Korrespondenzadresse}

Prof. Dr. R. Felberbaum

Klinik für Frauenheilkunde und Geburtshilfe, Perinatalzentrum, Zentrum für Reproduktionsmedizin, Klinikum Kempten - Oberallgäu Robert-Weixler-Str. 50, 87439 Kempten (Allgäu) Ricardo.Felberbaum@Klinikum-Kempten.de

Interessenkonflikt. Keine Angaben.
Abb. 1 - Triamniotedichoriale Drillingsgravidität

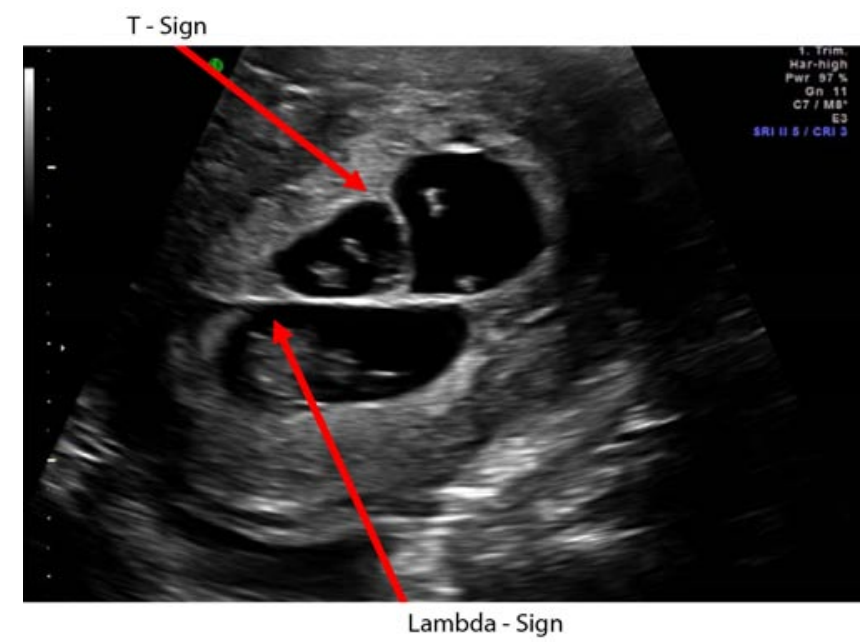

